\title{
Comparison of Temporal Conception between Chinese and English
}

\author{
Yujie $\mathrm{Su}^{1, *}$ \\ ${ }^{1}$ School of Humanities, Wenzhou University, Wenzhou, China \\ *Correspondence: School of Humanities, Wenzhou University, Wenzhou, China. E-mail: 424185224@qq.com
}

Received: September 10, 2018 Accepted: November 26, 2018 Online Published: January 17, 2019

doi:10.5430/sass.v6n1p11 URL: https://doi.org/10.5430/sass.v6n1p11

\begin{abstract}
Chinese and English are of variant language systems, which account for some differences in their temporal concepts. In terms of the temporal concepts EARLIER and LATER between Chinese and English, Vyvyan Evans and Melanie Green came to a conclusion that English tend to view time on a horizontal axis while Chinese on a vertical axis. The present research intends to obtain a more general and objective view of temporal difference across Chinese and English, by referring to definitions and examples in Chinese and English dictionaries and doing a survey among senior English majors in Wenzhou University.
\end{abstract}

Keywords: temporal concept, difference, Chinese, English

\section{Introduction}

Vyvyan Evans and Melanie Green proposed 3 main cognitive models for TIME, the moving time model, the moving ego model, the temporal sequence model. They explained that while the first two were ego-based and typically involved reference to the present or 'now', the third kind was time-based and made no intrinsic reference to the concept of 'now'. And they found there was a contrast between the English pattern and the Mandarin pattern in term of the temporal concepts EARLIER and LATER. The former was elaborated on the horizontal axis while the latter one was on the vertical axis, (2006: 84-94) as illustrated in the following examples:

(1) a. Tuesday comes/is before Wednesday.

b. Wednesday comes/ is after Tuesday.

(2) a. shang-ban-ye

上 半 夜 (upper-half-night: before midnight)

b. xia-ban-ye

下 半 夜 (lower-half-night: after midnight)

(Vyvyan Evans \& Melanie Green, 2006: 94-95)

Examples (1)-(2) correctly illustrate the distinction between English and Chinese. The examples (1a) and (1b) indicate English speakers tend to view time flowing from the past to the future, while (2a) and (2b) show Chinese speakers tend to conceive time in a vertical way.

But like Boroditsky(note 1)(2001), all of them were too hurry to draw the conclusion. Firstly, as for the phrases "shang-bei" and "xia-bei" in Examples (3a) and (3b), their meanings are not the same as a Chinese native speaker thought.

(3) a. shang-bei

上辈 (upper generation: the elder generation)

b. xia-bei

下 辈 (lower generation: the younger generation)

(Vyvyan Evans \& Melanie Green, 2006: 94-95) 
All of these examples such as (1a-b), (2a-b) and 3(a-b) in the book coauthored by Evans and Green are from the findings of Ning Yu (1999). More correctly speaking, "shang-bei" refers to a person who is of one's father or grandfather's age or social relation ranks. For example, my parents and grandparents are my elder generation, namely, "shang-bei-ren (people of the generation prior to one's own generation)". The other equivalent is "qian-bei" in Chinese. "hou-bei" is the opposite term for "qian-bei", meaning one's offspring or descendants. These expressions refer to people of certain generations or grades. But when interpreted as a temporal unit, "shang-bei" is equal to "shang-bei-zi" or "one's previous life".

Spatial terms like "qian/hou (front/ back)" are also commonly used in Chinese to refer to earlier or later time concepts. "qian-tian" refers to the day before yesterday while "hou-tian" is equivalent to the day after tomorrow in English. In addition, Spatiotemporal concepts in Chinese are not the same as many cognitive linguists (Clark, 1973; Boroditsky, 2000) have found to be one-dimensional, multi-dimensional.

Hence, some questions arise, such as how the temporal concepts EALIER and LATER are conceptualized in Mandarin or Chinese? Do Chinese conceptualize time horizontally, vertically or both? What are the other temporal concepts found in Chinese? What can the distinct temporal concepts reveal between Chinese and English? Only by finding solutions or answers to the questions above can a general and objective conclusion be drawn.

\section{Temporal Conception Researches}

Unlike space, time is not a concrete or physical sensor experience, but abstract and shapeless. Despite this, time can be conceived metaphorically in different languages, as Lakoff (1993: 218) stated that "The fact that time is understood metaphorically in terms of motion, entities, and location accords with our biological knowledge. In our visual systems, we have detectors for motions and detectors for objects/ location. We do not have detectors for time. Thus, it makes good biological sense that time should be understood in terms of things and motion." Kövesces (2010: 204) put forward that two simultaneous pressures brought forth metaphorical conceptualization in natural situations: the pressure of embodiment and the pressure of context, which both amounted to our effort to be coherent with the body and culture-coherent both with universal embodiment and the culture-specificity of local culture in the course of metaphorical conceptualization. All indicate to us that the idea that time is conceived in terms of spatial terms such as location, motion and other sources like entity has been well confirmed among cognitive linguists.

\subsection{Time in Terms of Location}

The researches on the metaphorical conceptualization of the abstract concept TIME by means of the concrete special terms have been done in a wide range of languages throughout the world (Boroditsky et al., 2010; Duffy \& Feist, 2014; Hsieh, 2013; Zhou \& Fan, 2015). By means of key-word searching, the Google Scholar engine located 114 results of "temporal-spatial metaphor" or "spatial-temporal metaphor", respectively 62 general linguistic theoretical studies, 30 cross-linguistic and 22 other scientific researches.(note 2) The results indicate a high tendency of temporal-spatial mappings in the related researches.

Lakoff (1993: 213) stated that many of the most basic concepts like time, quantity, etc, in our conceptual systems were normally comprehended via metaphor, and time in English is conceptualized in terms of space. For instance, English speakers prefer horizontal spatial terms to vertical ones in describing time. (Boroditsky, 2001; Evans \& Green, 2006) "Monday is before Tuesday." is more naturally accepted than "Monday is above Tuesday." Spatial terms such as preposition, adverbs are most commonly used for temporal representation. "Nearly every aspect of time can be expressed in spatial words: instants in time can be points; durations can be long or short; events can be moved forward or pushed back." (Casasanto \& Jasmin, 2012: 644)

"Although space has the same physical properties all around the world, the location of the human body and of objects within geographical confinements is encoded in different ways cross-linguistically" (Fulga, 2012: 27). English is not the only language to employ an ego-oriented perspective in spatiotemporal conceptualization. There was an orderly and systematic correspondence between the domains of time and space in language, such as uni-dimensional spatial terms like front/ back, up/ down borrowed to describe one-dimensional time, while two or three dimensional special terms like narrow/ wide, shallow/ deep were rarely borrowed (Gentner, 2001: 203). Chen \& Huang (2006) suggested in their research there were 3 kinds temporal modes: the linear mode, the circular modes, the spiral mode, in other terms, front/ back axis, left/ right axis, vertical axis or three axes (Zhang, 2003; Julio Santiago et. al., 2007; Li \& Wang, 2015).

The striking distinction between Chinese and English has been well established in cognitive literature that compared with English, Chinese usually applies the vertical axis in conceptualizing time (Ahrens \& Huang, 2002; Radden, 
2011; Yu 1998). But after the careful investigation into the Chinese dictionaries and the relative spatial-temporary academic researches, it turned out some misunderstandings should be corrected.

Table 1. Spatial Terms Used in Temporal Representation in Chinese and English

\begin{tabular}{|c|c|c|}
\hline spatial terms & Chinese & English \\
\hline shàng (up) & $\begin{array}{l}\text { shang-ce (up-volume: the first half } \\
\text { volume of a book); shang-qi } \\
\text { (up-period: last period) }\end{array}$ & 1 \\
\hline xià (down) & $\begin{array}{l}\text { xia-ce (down-volume: the second } \\
\text { half volume of a book); xia-qi } \\
\text { (down-period: next period) }\end{array}$ & l \\
\hline qián (front, before) & $\begin{array}{l}\text { qian-tian (front-day: the day before } \\
\text { yesterday); qian-tu (front-road: } \\
\text { future road of life) }\end{array}$ & Monday is before Wednesday. \\
\hline hòu (back, after) & $\begin{array}{l}\text { hou-tian (back-day: the day after } \\
\text { tomorrow); hou-xiao (back-effect: } \\
\text { the future effect) }\end{array}$ & Wednesday is after Tuesday. \\
\hline yuán (far) & $\begin{array}{l}\text { yuan-ri (far-day: the day far in the } \\
\text { future) }\end{array}$ & far into the night; as far back as 1949 \\
\hline jìn (near) & $\begin{array}{l}\text { jin-qi (near-period: the recent days or } \\
\text { period) }\end{array}$ & the near future \\
\hline shēn (deep) & shen-ye (deep-night: late at night) & I \\
\hline qián (shallow) & ri-zi-qian (day-shallow: a short time) & I \\
\hline chăng (long) & chang-qi (long-period: long term) & for a long time \\
\hline duăn (short) & duan-qi (short-period: short term) & for a short time \\
\hline zuó-yòu (left-right) & $\begin{array}{l}\text { only used in combination to modify } \\
\text { number, meaning "about, around, or } \\
\text { so", equal to "shang-xia (up-down)" }\end{array}$ & / \\
\hline shàng-xià (up-down) & $\begin{array}{l}\text { to modify number, meaning "about, } \\
\text { around, or so", similar to } \\
\text { "zuo-you(left-right)" }\end{array}$ & l \\
\hline
\end{tabular}

Table 1 shows that both English and Chinese have conceived time in terms of location and duration of time. And there is no vertical temporal conception in English, but in Chinese, besides the vertical temporal conception, there are other temporal conceptions in reference to the depth of time. Apart from that vertical spatial terms like "upper" or "up" can be used in Chinese temporal conception, other dimensional spatial terms like "shen/qian (deeper/ shallower)", "zuo-you (left-right)", "yuan/jin (far/near)", "chang-duan (long/short)", etc, are also optional for temporal conception in Chinese. For example, the night is usually scaled in terms of depth, such as "shen-ye (deep night: late at night)" and "ye-shen-ren-jin (night deep people quiet: the still of night)", while "ri-zi-qian (days shallow: not long)" is specifically used to mean that a woman has been pregnant for a short time. And the era existing long long time ago is referred to be "yuan-gu (far ancient: ancient times)", while the days close to the present time is often called as "jin-ri (near day: recently)". In addition, time can be scaled as long or short, such as "chang-shi-jian (long time)" and "duan-shi-jian (short time)". When the spatial terms like "zuo" or "you" are used to refer the temporal concepts, both are combined together, forming an adverb phrase of degree "zuo-you". It is equal to "shang-xia", which can be used in the representation of time and other numerals, meaning "about, around, or so".

(4) a. shi-ge-yue shang-xia de ying-er

$$
\text { 十个月上下的婴儿 (a baby of about ten months old) }
$$

b. nian-ji zai san-shi-sui zuo-you 


\section{年 纪在三十 岁 左 右 (about 30 years old)}

In (4) a and b, it can be seen that both the vertical spatial term "shang-xia" and horizontal spatial term "zuo-you" are used in the temporal domain.

Comparatively speaking, English spatiotemporal representations are not as variable as Chinese counterparts. Chinese temporal conception is of a multi-axis system, in this sense. In Chinese, there is a hierarchical rank among the axes, the vertical temporal conception followed by horizontal or left/ right axis, or front/ back axis, which might be what Boroditsky (2011: 64) the result of influences of writing direction on how we organized time.

\subsection{Time in Terms of Motion}

According to Evans (2004: 78-79), temporal experience, as it is represented and encoded in language, exhibits two levels of organization, lexical concepts and cognitive models for time. For example, we may think of time moving back or forth in English and up or down in Chinese. According to figure-scene schema of the moving time model, the time is a figure and the ego is the scene, while in the moving ego model, the ego is a figure and the time becomes the scene. Some cognitive linguists (Yu, 1998; Ahrens \& Huang, 2002) interpreted it as the conception THE PASSING TIME IS MOTION metaphor. There are two space-time metaphoric systems or modals: the ego-moving metaphor and the time-moving metaphor (Evans \& Green, 2006; Gentner et al., 2002; Lakoff \& Johnson, 1980). That is identical to what Lakoff (1992) has put forward as TIME PASSING IS MOTION OF AN OBJECT and TIME PASSING IS AN OBSERVER'S MOTION OVER A LANDSCAPE.

In English future times are in front of the observer and past times are behind the observer, while for a Chinese native speaker, generally past times are in front of the observer and future time are behind the observer. In other words, English and Chinese tend to take a distinct biological locality on the path of time, which can be seen clearly in terms of "qian/hou" temporal representation.

But in terms of motion pattern, Chinese and English take the similar temporal conceptualization, for example in the works of a distinguished Chinese writer Ziqing Zhu, a majority of such examples:

Thus the day flows away through the sink when I wash my hands; vanishes in the rice bowl when I have my meal; passes away quietly before the fixed gaze of my eyes when I am lost in reverie. Aware of its fleeting presence, I reach out for it only to find it brushing past my outstretched hands. In the evening, when I lie on my bed, it nimbly strides over my body and flits past my feet. By the time when I open my eyes to meet the sun again, another day is already gone. I heave a sigh, my head buried in my hands. But, in the midst of my sighs, a new day is flashing past.(note 3)

The words of motions presented in italics in Zhu's writing show that in Chinese e passage of time is also often conceived as the flow of water, or the strides of a person, or the flashes of light.

\subsection{Time in Terms of Entity}

Time can be mapped into the domain of ENTITY. Metaphors like "TIME IS MONEY", "TIME IS A JOURNEY", etc, are abundant in all cultures. Ungerer \& Schmid (1996: 118) assumed the target category TIME via the source category MONEY, i.e. a valuable commodity and limited source in the following English phrases:

You're wasting my time.

Can you give me a few minutes?

How do you spend your time?

We are running out of time.

Is that worth your while?

In Chinese, there is also a TIME IS MONEY metaphor, but the underlying cultural motivation is different from English (Huang, 2006; Yang, 2010). There are several explanations for the difference: 1) One lies in the fact that the ancient Chinese paid much attention to the heavenly bodies and their movements, such as the movements of the sun, the moon and the stars because of the eternal features of their movements. In other words, the ancient Chinese observed that the period of the emergence of the sun was the day while the period of the emergence of the moon means the night. Based on the experiential observation, time is equal to "guang-yin (光阴 light shade or sun and moon)", which means the sun and moon. 2) Another unique experience of time in the Chinese culture is that "the Chinese calendar does not count years in an infinite sequence. Instead years have names that are repeated every 60 years. Within 60-year cycle, each year is assigned name consisting of two component: a Celestial Stemm (甲乙丙丁 戊已庚辛壬癸) and a Terrestrial Branch (the names of the corresponding animals in the zodiac cycle of 12 animals: 
rat, ox, tiger, hare, dragon, snake, horse, sheep, monkey, rooster, dog and pig)"(note 4). As for the idiom “hou-nian-ma-yue (猴年马月 monkey- year- horse- month)", it appears once every 12 years according to the lunar calendar, which is not a short time span. And it is said that a prophecy was related to the idiom that one day in the far future a momentous event would take place. In general, the idiom now means an unknown year in the future, equal to the English idiom: till the cows come home(note 5), which does not appear in the Chinese vocabulary. 3) The ancient China relied heavily on agriculture, which generated a temporal concept that time was short and precious. The farmers or peasants used to describe time passage in terms of the alternate variations of the sun and the moon. Hence, there is a fortune of famous temporal saying in Chinese, such as "shi-jian shi guang-yin (时间是光阴 Time is sunlight and moonlight)", “yi-cun-guang-yin yi-cun-jin (一寸光阴一寸金 literarily translated as "an inch of sunlight is worth an inch of gold", while well-accepted one "Every second counts" or "Time is gold"or "Time is money". Both indicate the truth that time is transient and short, and time is precious. Through the process of conceptualization, the domain of time is mapped into the domain of money, generating the concepts like "time can be spent, wasted, saved, planned and owned like money”, which are sufficient in examples such as “花时间 (spend time)”, “浪费时间 (waste time)”, “挤时间 (spare time)”, “赢得时间(win time)”, “珍惜时间(cherish time)”, “争分夺秒(race against time, make the best use of time)", and so on. "the universal bodily basis on which the universal metaphors could be built is not utilized in the same way or to the same extent in different languages or varieties of languages," due to the different experiential focus in mind (Kövecses, 2005:246; 2006: 2-3).

\section{Conclusion}

By contrast, both English and Chinese have more differences than similarities in their temporal conception, in location, motion and eternity. In English, time is more likely to be conceived as a horizontal sequence, while in Chinese, time can be horizontal, vertical and multidirectional. The difference in temporal conception between the two languages results in their distinct cultural background. One arises from the ancient worship of the sun and the planets alike. The other one originates from the Chinese ontological philosophy based on the ancient Chinese calendar and agriculture. Thus, the Chinese time has much more flexible conception than the English one.

The present paper presents the similarities and differences between English and Chinese on the basis of linguistic representations. Whether the different temporal conception may influence the behavior can be the following topic in future.

\section{References}

Ahrens, Kathleen \& Chu-Ren Huang. (2002). Time Passing is Motion. Language and Linguistics, 3(3), 491-519.

Boroditsky, L. (2000). Metaphoric structuring: understanding time through spatial metaphors. Cognition, 75, 1-28. https://doi.org/10.1016/S0010-0277(99)00073-6

Boroditsky, L. (2001). Does Language Shape Thought? Mandarin and English Speakers' Conceptions of Time. Cognitive Psychology, 43, 1-22. https://doi.org/10.1006/cogp.2001.0748

Boroditsky, L. (2011). How Language Construct Time. In Stanislas Dehaene \& Elizabeth M. Brannon (Eds.), Space, Time and Number in the Brain: Searching for the Foundations of Mathematical Thought (pp.333-341). London: Elsevier Inc. https://doi.org/10.1016/B978-0-12-385948-8.00020-7

Boroditsky, L., Fuhrman, O., \& K. McCormick. (2010). Do English and Mandarin Speakers Think about Time Differently? Cognition, 9(10), 1-7.

Casasanto, Daniel \& Kyle Jasmin. (2012). The Hands of Time: Temporal gestures in English speakers. Cognitive Linguistics, 23(4), 643-674. https://doi.org/10.1515/cog-2012-0020

Chen, Yan \& Huang, Xi-ting. (2006). A Review on Time Metaphor. Advances in Psychological Science, 14(4), 604-609.

Duffy, Sarah. E., \& Michele L. Feist. (2014). Individual Difference in the Interpretation of Ambiguous Statements about Time. Cognitive Linguistics, 25(1), 29-54. https://doi.org/10.1515/cog-2013-0030

Evans, Vyvyan \& Melanie Greens. (2006). Cognitive Linguistics: An introduction. New Jersey: Lawrence Erlbaum Association, Inc. Publishers.

Evans, Vyvyan. (2004). The Structure of Time: Language, Meaning and Temporal Cognition. Amsterdam: John Benjamins. https://doi.org/10.1075/hcp.12 
Fulga. Angelica. (2012). Language and the perception of Space, Motion and Time. Concordia Working Papers in Applied Linguistics, 3, 26-37.

Gentner, D., Imai, Mutsumi \& Lera Boroditsky. (2002). As time goes by: Evidence for two systems in processing space $\rightarrow$ time metaphors. Language and Cognitive Processes, 17(5), 537-565. https://doi.org/10.1080/01690960143000317

Gentner, D. (2001). Spatial Metaphors in Temporal Reasoning. In M. Gattis (Ed.), Spatial Schemas in Abstract Thought (pp. 203-222). Cambridge, MA: MIT Press.

Hsieh, Shelley Ching-yu. (2013). Hyperbole of Time \& Space in Three Languages. Intergrams, 13(2), 1-18.

Huang, Yu-lan. (2006). A Comparative Study of Metaphor in English and Chinese Proverbs about Time. Science and Technology Consulting Herald, 14, 150-151.

Julio Santiago, Juan Lupiáñez, Elvira Pérez \& María Jesús Funes. (2007). Time (also) Flies from Left to Right. Psychonomic Bulletin \& Review, 14(3), 512-516. https://doi.org/10.3758/BF03194099

Kathleen Ahrens \& Chu-Ren Huang. (2002). Time Passing Is Motion. Language and Linguistics, 3(3), 491-519.

Kövesces, Zoltán. (2010). Metaphor and Culture. Acta Universitatis Sapientiae, Philologica, 2(2), 197-220.

Lakoff, G. (1993). The Contemporary Theory of Metaphor. In Andrew Ortony (Ed.), Metaphor and Thought (pp.202-251). Cambridge: Cambridge University Press. https://doi.org/10.1017/CBO9781139173865.013

Lakoff, G., \& M. Johnson. (1980). Metaphors we live by. Chicago: University of Chicago Press.

Li, Jin-xing \& Wang, Zhen-hong. (2015). Spatial-temporal Association of Response Codes Effect: Manifestation, Influencing Factors and Its Theories. Advances in Psychological Science, 23(1), 30-40. https://doi.org/10.3724/SP.J.1042.2015.00030

Radden, Günter. (2011). Spatial Time in the West and the East. In Maria Brdar, Marjija Omazic, Visnija pavicic Takac, Tanja Gradecak-Erdeljic, and Gabrijela Buljan (Eds.), Space and Time in Language (pp.1-40). Frankfurt et al.: Peter Lang.

Ungerer, Friedrich \& Hans-Jörg Schmid. (1996). An Introduction to Cognitive Linguistics. New York: Addison Wesley Longman Limited.

Yang, Mei. (2010). A Cognitive Study of Temporal Metaphors in Chinese. Journal of Zhaotong Teacher's College, 32(1), 16-19.

Yu, Ning. (1998). The contemporary theory of metaphor: A perspective from Chinese. Amsterdam: John Benjamins Publishing Company. https://doi.org/10.1075/hcp.1

Zhang, Jian-li. (2003). Conceptualization and Expression of qian and hou in Chinese Temporal System. Journal of Zhejiang University (Humanities and Social Sciences), 33(5), 84-91.

Zhou, Yanchun \& Yanhong, Fan. (2015). A Contrastive Study of Temporal-spatial Metaphor between Chinese and American. Theory and Practice in Language Studies, 5(1), 119-123. https://doi.org/10.17507/tpls.0501.16

\section{Notes}

Note 1. Boroditsky (2001: 18-19) had ever generated in the research by comparing the time native English and native mandarin speakers responded to the questions phrased in purely temporal earlier/ later terms, that the fact that Mandarin speakers commonly used the vertical terms to talk about time predicted that they were more natural to construct a vertical time line when thinking about purely temporal relations, while English speakers tend to think about time horizontally because horizontal spatial terms predominated in English temporal descriptions.

Note 2 . The results are generated by language-specific searching mode. Two languages are required. The data were obtained on March 17, 2018.

Note 3. http://www.hjenglish.com/fanyi/p477418/. March 26, 2016.

Note 4. http://www.webexhibits.org/calendars/calendar-chinese.html. Accessed on March 29, 2016.

Note 5. http://www.manmankan.com/nizhidaoma/article/2015/159.html. Accessed on March 29, 2016. 